\section{Hilfe bei Juckreiz und Quaddelbildung}

D as seit Februar auf dem deutschen Markt erhältliche Antihistaminikum Levocetirizin hat zuverlässige und langanhaltende Erfolge bei akuter und chronischer Urtikaria sowie antientzündliche Eigenschaften gezeigt, so A. Kapp, Hannover. „Ein Präparat, das zur Behandlung bei Urtikaria eingesetzt wird, muss erstens den quälenden Juckreiz lindern, und darüber hinaus sollten die Quaddeln verschwinden", sagte Kapp. Bei klinischen Tests an gesunden Probanden mit histamininduzierten Hautreaktionen habe der Juckreiz mit Levocetirizin nahezu auf Null reduziert werden können. Des weiteren sei es zu einer 95prozentigen Hemmung der Quaddelbildung gekommen.

Diese Resultate habe er nicht erwartet, erklärte Kapp. Wirkung, Wirk- dauer und Zuverlässigkeit von Levocetirizin habe sich gegenüber einer Reihe bekannter Antihistaminika überlegen gezeigt. Kapp: „Dies ist das beste inhibitorische Antihistaminikum, das bisher getestet worden ist."

\section{Für alte Menschen verträglich}

Besonders geeignet sei das Medikament auch bei chronischer Urtikaria, die häufig bei Patienten in höherem Lebensalter auftrete. „Diese Patienten brauchen eine Therapie über Wochen und Monate", sagte Kapp. Da bei alten Menschen oft andere Erkrankungen vorliegen, die medikamentös behandelt werden müssen, sei es wichtig, eine Substanz zu haben, die kaum Nebenwirkungen hat und kaum metabolisiert wird, um Wirkungsinterferenzen zu vermeiden. Dies treffe auf Levocetirizin zu. Die Rate an unerwünschten Wirkungen liege im Plazebo-Bereich.

Erste Untersuchungen der antientzündlichen Eigenschaften von Levocetirizin ergaben nach Angaben von Kapp ebenfalls positive Ergebnisse. So kam es bei 14 Probanden mit Graspollen-Allergie, denen das Allergen per Hautkammertechnik appliziert worden war, zu einer signifikanten Hemmung der Eosinophilen-Migration ins Entzündungsgebiet.

Kapps Fazit: Levocetirizin $5 \mathrm{mg}$ täglich ist mindestens genauso wirksam wie Cetirizin $10 \mathrm{mg}$ täglich. Die Antihistamin-Wirkung sei stark, schnell und zuverlässig bei zusätzlich nachgewiesenen antientzündlichen Eigenschaften.

tm

RheinGAU-Symposium „Xusal ${ }^{\circledR}$ - Die neue Cetirizin-Generation“, 2001 Assmannshausen. Veranstaltet von UCB Pharma.

\section{Allergie auf Naturlatex - problematische Diagnostik}

$\mathrm{R}_{\mathrm{g}}$ und $1-10 \%$ der Bevölkerung sind gegen Naturlatex sensibilisiert. Gefahr droht vor allem bei medizinischen Eingriffen, so R. Brehler, Münster. Dies trifft in besonderem Maße auf mehrfach operierte Kinder zu, z.B. Kinder mit Spina bifida. Der präoperativen Diagnostik dieser Typ-I-Allergie kommt daher ein wichtiger Stellenwert zu, wobei die Vorgehensweise auf den klassischen Säulen Anamnese, Hauttest, In-vitro-Untersuchungen und Provokationstest ruht.

\section{Hauttest: Verschiedene Extrakte}

Beim Pricktest sollte man verschiedene Extrakte verwenden, da es bislang keinen kommerziellen Test gibt, der alle Latexallergiker gleich gut erfasst. Bewährt hat sich beispielsweise eine „high ammoniated" Latexmilch, die Handschuhhersteller zur Verfügung stellen.

Auch die 30-minütige Extraktion von Latexhandschuhen in physiologischer Kochsalzlösung bringt gute Ergebnisse. Allerdings schwankt der Pro- tein- und Allergengehalt von Fabrikat zu Fabrikat massiv, so dass entweder verschiedene Handschuhmarken oder speziell die an der Klinik verwendeten Handschuhe getestet werden müssen. Auch von allergologisch spezialisierten Firmen sind jetzt zumindest für Studienzwecke fertige Extrakte erhältlich.

\section{In vitro: Cave Kreuzreaktion}

Für den Nachweis von spezifischem IgE gegen Naturlatex kann man auf die Systeme verschiedener Hersteller zurück greifen. Die Sensitivität schwankt allerdings erheblich und wird in der Literatur mit Werten von 25-95\% angegeben. Die Spezifität dürfte zwischen 90 und $95 \%$ liegen. Ein Hauptproblem bei diesen Testungen sind die falsch positiven Resultate, d.h. es wird spezifisches Latex-IgE nachgewiesen, ohne dass sich bei den Patienten in Anamnese oder Pricktest eine Latexallergie eruieren lässt. Ursache für die falsch positiven Ergebnisse dürften vor allem Kreuzreak- tionen zu bestimmten Früchten oder Pollen sein, deren Allergene ausgeprägte Strukturhomologien zu Latexallergenen aufweisen. Nachgewiesen wurde dies, so X. Baur, Hamburg, für Avocados, Bananen sowie Birkenpollen. Auch für Kiwi, Papaya, Paprika, Kartoffeln und Tomaten sind Kreuzreaktionen wahrscheinlich.

\section{Provokation: Resultat variiert}

Provokationstestungen, sonst Goldstandard in der Allergiediagnostik, sind bei Naturlatexallergie ebenfalls problematisch. Auch hier sind die Resultate stark von den eingesetzten Handschuhen mit ihrem unterschiedlichen Proteingehalt wie auch vom Testprotokoll abhängig. Die Sensitivität wird mit Werten zwischen etwa 60 und 90\% angegeben.

Vor diesem Hintergrund, so Brehler, ist für den sicheren Nachweis einer Latex-Allergie der Hauttest das beste Verfahren. Wichtig aber: Um eine Allergie wirklich ausschließen zu können, müssen immer mehrere Extrakte verwendet werden.

Satellitensymposium der Allergopharma Joachim Ganzer KG anlässlich des 26. ÄDA-Kongresses, Bonn, 2000. 